index ocomunicación | no 10(3) 2020 | Páginas 215-233

E-ISSN: 2174-1859 | ISSN: 2444-3239 | Depósito Legal: M-19965-2015

Recibido el 22_09_2020 | Aceptado el 06_10_2020 | Publicado el 12_11_2020

\title{
EDUCOMUNICACIÓN Y 'MEDIA LITERACY': ESPACIOS DE REFERENCIA EN DIVULGACIÓN CIENTÍFICA Y ACADÉMICA EN ESPAÑOL
}

\author{
EDUCOMMUNICATION AND 'MEDIA LITERACY': \\ SPACES OF REFERENCE IN SCIENTIFIC \\ AND ACADEMIC DIFFUSION IN SPANISH
}

https://doi.org/10.33732/ixc/10/03Educom

Ignacio Aguaded

Universidad de Huelva aguaded@uhu.es https://orcid.org/0000-0002-0229-1118

\section{Bárbara Castillo-Abdul}

Universidad de Huelva

barbaracastilloabdul@gmail.com

https://orcid.org/0000-0002-3711-1519

\section{Luis M. Romero-Rodríguez Universidad Espíritu Santo (Visiting Professor) luis@romero-rodriquez.com https://orcid.org/0000-0003-3924-1517}

Este trabajo se enmarca en 'Alfamed' (Red Euroamericana de Investigadores), con el apoyo del Proyecto I+D «YOUTUBERS E INSTAGRAMMERS: LA COMPETENCIA MEDIÁTICA EN LOS PROSUMIDORES EMERGENTES» (RTI2018-093303-B-I00), financiado por la Agencia Estatal de Investigación del Ministerio de Ciencia, Innovación y Universidades de España y el Fondo Europeo de Desarrollo Regional (FEDER)

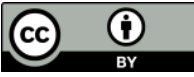

Para citar este trabajo: Aguaded, I., Castillo-Abdul, B. y RomeroRodríguez, L.M. (2020). Educomunicación y Media Literacy: espacios de referencia en divulgación científica y académica en español. index.comunicación, 10(3), 215-233. https://doi.org/10.33732/ixc/10/03Educom 
Resumen: La presente investigación realiza una revisión pormenorizada de los principales espacios de divulgación de investigaciones sobre educomunicación y media literacy en Iberoamérica, tomando como referencia revistas de alto impacto (JCR/Scopus), publicaciones en índices emergentes (ESCI), publicaciones históricas de reconocida trayectoria y congresos de referencia internacional. En el ámbito de las revistas científicas de alto impacto se evidencian espacios reducidos para la publicación en español, lo que deviene en altas tasas de rechazo. Sin embargo, algunas revistas del Emerging Sources Citation Index (ESCI) han mejorado su posicionamiento relativo, cogiendo el testigo de la temática de educomunicación. Asimismo, se revisan algunas publicaciones históricas de la comunicación y la educación, como la Revista Comunicar, especialista en la materia, Chasqui, Estudios Venezolanos de la Comunicación, Contratexto y Comunicação \& Educação, algunas con más de 50 años de existencia. Se concluye con una enumeración de los principales congresos internacionales de la temática, para ofrecer, en definitiva, una especie de mapping sobre los principales espacios de difusión de esta temática en nuestro idioma.

Palabras clave: educomunicación; media literacy; comunicación; educación; divulgación científica; publicaciones.

Abstract: The present research carries out a detailed review of the main spaces for disseminating research on educommunication and media literacy in the Iberoamerican space, taking as a reference high impact journals (JCR/Scopus), publications in emerging indexes (ESCI), historical publications of recognized trajectory and congresses of international reference. In the field of high-impact scientific journals, there is evidence of reduced space for publication in Spanish, which results in high rejection rates. Nevertheless, some journals of the Emerging Sources Citation Index (ESCI) have improved their relative positioning, taking the baton from the educommunication thematic. Likewise, some historical publications of communication and education are reviewed, such as Comunicar Journal, a specialist in the subject, Chasqui Journal, Estudios Venezolanos de la Comunicación, Contratexto and Comunicação \& Educação, some of them with more than 50 years of existence. It concludes with a list of the leading international congresses on the subject of education and communication, to offer, in short, a kind of mapping on the spaces of dissemination of this topic in Spanish.

Keywords: Educommunication; Media literacy; Communication; Education; Scientific dissemination; Publications. 


\section{Introducción}

Difundir los resultados de investigaciones sobre educomunicación en nuestro idioma no resulta una tarea fácil, fundamentalmente por los restringidos - ergo, colapsados - espacios que existen para ello (Castillo y Carretón, 2010; De-Filippo, 2013; Mancinas-Chávez, Romero-Rodríguez y Aguaded, 2016). Quizás, la primera de las dificultades que afrontamos es la delimitación epistemológica del campo (Piñeiro-Naval y Morais, 2019; RomeroRodríguez y Aguaded, 2016) y la comprensión de su transdisciplinariedad pues, mientras que para algunas publicaciones del ámbito de la comunicación la educomunicación pertenece al campo de la educación, para sus homólogas de educación, ésta pertenece a la delimitación de la comunicación.

En esta línea, la investigación de Lotero-Echeverri, Romero-Rodríguez y Pérez-Rodríguez (2019), en la que se realiza un análisis pormenorizado de 13 revistas científicas iberoamericanas indexadas en Scopus en 2018, coincide con Mancinas-Chávez et al. (2016) en que, dado el crecimiento de requerimientos a los profesores de artículos publicados en revistas indexadas por parte de agencias de acreditación de varios países iberoamericanos ${ }^{1}$ — siguiendo la lógica del publish or perish - y el nulo o muy lento ingreso a bases de datos selectivas (Web of Science-JCR y Scopus) de publicaciones que publiquen, o al menos evalúen, manuscritos en español, las pocas revistas que lo admiten se encuentran colapsadas, pero además, intentando luchar contra la endogamia regional que afecta su posicionamiento e impacto en las bases de datos precitadas.

No obstante comprender que la lengua franca de la investigación es por antonomasia el inglés, no es menos cierto - ni arriesgado- asegurar que son muy pocos los investigadores iberoamericanos del campo de la educomunicación que dominan este idioma a la perfección, con capacidad suficiente para presentar un manuscrito con la calidad de redacción necesaria para una revista científica de impacto. En este sentido, para nadie es un secreto que, además de tener que utilizar los escasos recursos económicos para la realización de la investigación, los autores iberoamericanos han de tener también recursos para financiar la traducción profesional, o al menos el servicio de edición profesional en idioma inglés, lo que ensancha aún más las brechas de limitaciones con nuestros pares norteamericanos, británicos y del resto de Europa (Berger y Chafee, 2006).

Si bien el problema idiomático no es el único que no permite conservar la investigación en educomunicación en Iberoamérica en consonancia con el tratamiento que ésta tiene en otras regiones de mayor desarrollo científico,

${ }^{1}$ Principalmente: CONACYT [México], COLCIENCIAS [Colombia], SENESCYT [Ecuador], CAPES [Brasil], ANECA y CNEAI [España] y CONICYT [Chile]. 
sobre todo por los grandes inconvenientes de formación en metodología de investigación en universidades latinoamericanas (González-Samé, 2019; González-Samé, Romero-Rodríguez y Aguaded, 2017), sí constituye un factor decisivo para los resultados cuantitativos de documentos publicados en bases de datos selectivas. De hecho, de los 45.974 documentos emergentes en Scopus por el criterio de búsqueda [«Media*» AND «Education*»]2, solo $1136(2,47 \%)$ tienen versión en español, siendo estos publicados principalmente en las siguientes revistas:

Figura 1. Publicaciones en español indexadas en Scopus con artículos que contienen metadatos sobre educomunicación (Media Education)

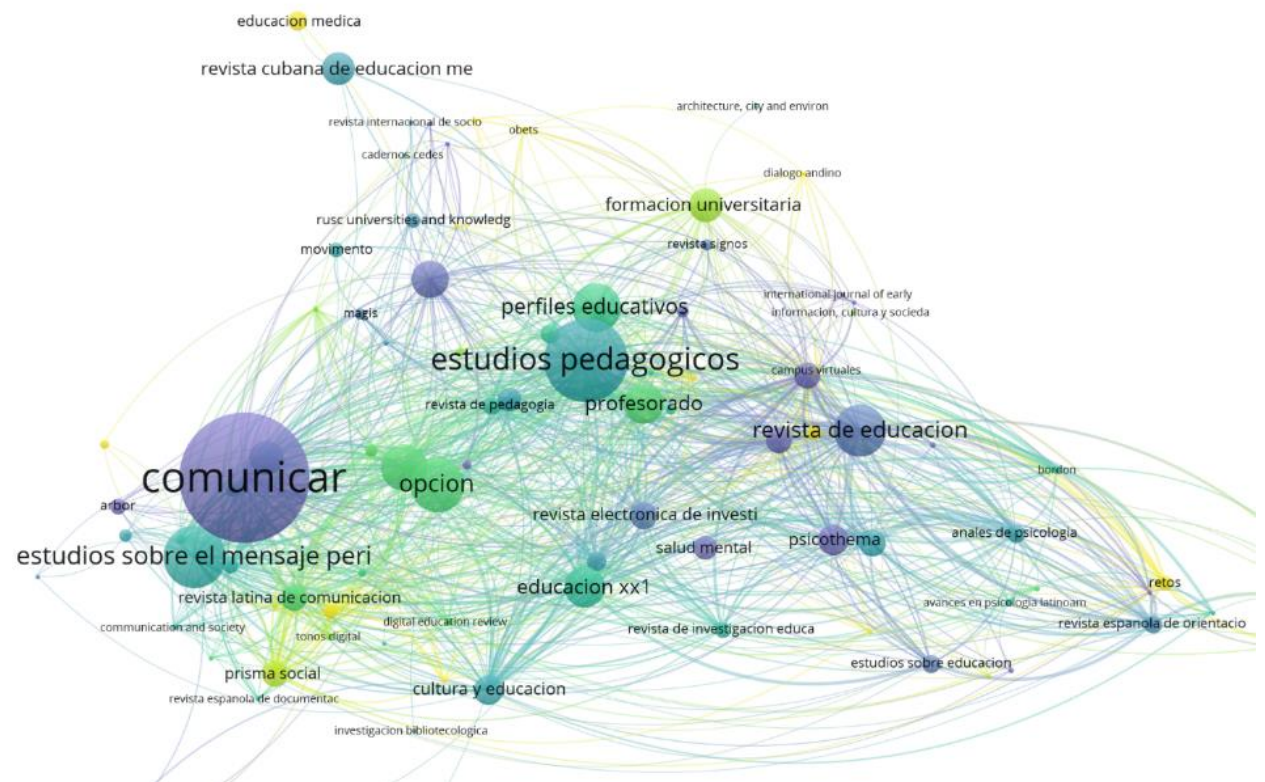

Fuente: elaboración propia.

Como se extrae de la figura anterior, la Revista Comunicar ${ }^{3}$ mantiene una posición central e histórica en la temática, siendo una publicación que se ha posicionado muy bien dentro de los índices de impacto selectivo, pero además especialista en la materia, lo que le permite ser núcleo de co-citación para sus pares iberoamericanas como Estudios sobre el Mensaje Periodístico,

2 Esta búsqueda fue realizada el 29/11/2019, limitando además los documentos emergentes a las áreas de Ciencias Sociales (Social Sciences) y Psicología (Psychology), tipo de documento [artículo].

3 Disponible en línea: https://www.revistacomunicar.com/ (Fecha de consulta: 29/11/2019). 
Educación XX1, Profesorado, Revista de Educación y Cultura y Educación (España), Universitas Psychologica (Colombia), Opción (Venezuela) y Estudios Pedagógicos (Chile).

Un ejercicio que nos permite analizar la centralidad temática de las revistas científicas es examinar las relaciones de citación (Gómez, Fernández y Sebastián, 1999). En este sentido, tomando como universo los 45.974 documentos emergentes en Scopus de la búsqueda anterior, se realiza una matriz de co-ocurrencia por citas. A diferencia con el revisado ut supra, este ejercicio se realiza con los documentos en todos los idiomas y no solo en español (ver figura 2).

\section{Figura 2. Matriz de co-ocurrencia de cohesión por citas de artículos indexados en Scopus con metadatos sobre educomunicación (Media Education)}

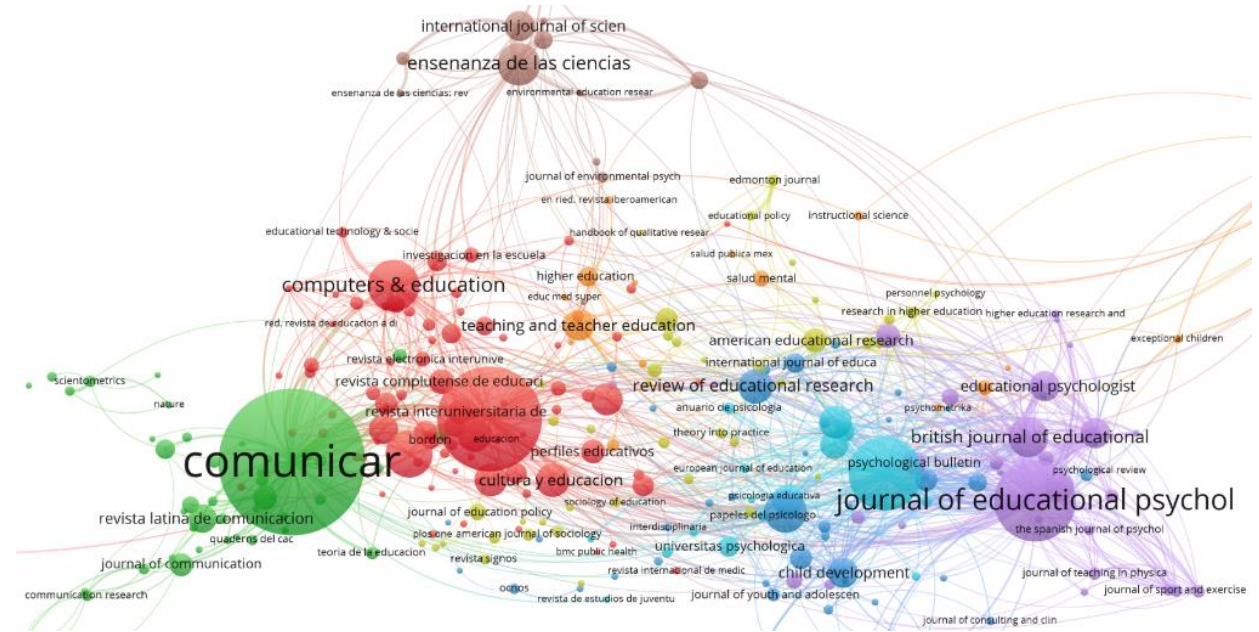

Fuente: elaboración propia.

Se evidencia además que en el caso de las revistas, a pesar de que Comunicar y Revista de Educación mantienen un papel central y con una densidad mucho mayor que otras, la mayoría de los artículos emergentes citados provienen de revistas «anglo» reconocidas en el ámbito de la educación y la psicología, como Computers \& Education, Journal of Educational Psychology, Review of Educational Research, Teaching and Teacher Education o Child Development. En esta línea, podemos extraer de la figura 2 el siguiente análisis:

1. La mayoría de las citas de los artículos sobre educomunicación (Media Education) son de documentos publicados en revistas de educación, lo que puede estar inclinando la balanza hacia la delimitación temática y epistemológica en este campo de estudio. Por supuesto, esto com- 
prendiendo que el número de revistas indexadas en Scopus de la categoría Communication (320) es más de tres veces menor que su par Education (1044).

2. La mayoría de las citas provenientes de Comunicar se hacen a revistas en español, como Psicothema, Infancia y Aprendizaje, Cultura y Educación, Enseñanza de las Ciencias, Estudios sobre el Mensaje Periodístico y Revista Latina de Comunicación Social. Esto indica, por una parte, que Comunicar está sirviendo como soporte de la investigación en educomunicación en español, apoyando la estabilidad de otras revistas iberoamericanas en las indexaciones, pero, por otra parte, que existe cierta «burbuja idiomática» por parte de los autores, bien por la incapacidad de buscar materiales en inglés y otros idiomas, o bien por el nexo cultural y ámbito geográfico de las investigaciones.

Por supuesto, difundir resultados sobre educomunicación va más allá de pensar en revistas científicas indexadas, pues también hay todo un universo de espacios en revistas especializadas indexadas en otras bases de datos, congresos y reuniones científicas de referencia, que posibilitan la socialización y difusión de nuestras investigaciones y, sobre todo, la creación de comunidades científicas sólidas.

\section{Publicaciones JCR y Scopus}

Las revistas incluidas en estas bases de datos son la pièce de résistance de las publicaciones científicas, pues se entiende con su indexación, por una parte, que tienen un recorrido histórico en una línea temática específica y, por otra, que son leídas y citadas por la comunidad científica internacional. Esto no quiere decir que las revistas no incluidas en estas bases de datos no sean importantes o de calidad, ni mucho menos que carecen de valor, pues hay revistas muy buenas no indexadas, mientras que hay revistas «deficientes» y con malas prácticas indexadas. En este orden de ideas, el estar o no indexadas es un indicador de que una revista es referencia internacional por el número de citas obtenido en una base de datos, pero no es el único indicador de su valor. Se debe insistir en que una publicación entra en estos índices selectivos porque ha obtenido una cantidad de citas en revistas que se encuentran en estos mismos índices, por lo cual el llamar a estas revistas como «de impacto» significa que son leídas, tienen buen alcance, son fuente de consulta y, en definitiva, logran posicionarse cuantitativamente en uno de estos exclusivos espacios.

a) Journal Citation Reports. En primer lugar, en el Journal Citation Reports (Clarivate Analytics), en su edición 2018, hay 88 revistas en la categoría Communication $\left(\mathrm{IF}_{\mathrm{m}}=1,495\right)$ y 243 en la categoría Education \& Educatio- 
nal Research $\left(\mathrm{IF}_{\mathrm{m}}=1,479\right)$, de las cuales en Communication solo hay dos españolas: Comunicar (Q1) y El Profesional de la Información (Q2), mientras que en Education \& Educational Research se posicionan 10 iberoamericanas (ver tabla 1 ):

Tabla 1. Revistas Iberoamericanas de la categoría Education \& Educational Research del Journal Citation Reports (2018)

\begin{tabular}{|l|l|c|c|}
\hline \multirow{5}{*}{ País } & \multicolumn{1}{|c|}{ Revista } & IF & Cuartil \\
\hline \multirow{5}{*}{ España } & Comunicar & 3,338 & Q1 \\
\cline { 2 - 4 } & $\begin{array}{l}\text { International Journal of Educational Technology } \\
\text { in Higher Education }\end{array}$ & 1,922 & $\mathrm{Q} 2$ \\
\cline { 2 - 4 } & Psicología Educativa & 1,613 & $\mathrm{Q} 2$ \\
\cline { 2 - 4 } & Educación XX1 & 1,597 & $\mathrm{Q} 2$ \\
\cline { 2 - 4 } & Revista Española de Pedagogía & 0,863 & $\mathrm{Q} 4$ \\
\cline { 2 - 4 } & Enseñanza de las Ciencias & 0,814 & $\mathrm{Q} 4$ \\
\cline { 2 - 4 } & Porta Linguarum & 0,506 & $\mathrm{Q} 4$ \\
\cline { 2 - 4 } & Revista de Educación & 0,310 & $\mathrm{Q} 4$ \\
\hline \multirow{2}{*}{ México } & $\begin{array}{l}\text { Revista Latinoamericana de Investigación } \\
\text { en Matemática Educativa (RELIME) }\end{array}$ & 0,950 & $\mathrm{Q} 4$ \\
\hline Brasil & Movimento & 0,307 & $\mathrm{Q} 4$ \\
\hline
\end{tabular}

Fuente: elaboración propia.

De la tabla anterior se extrae que de las 10 revistas iberoamericanas indexadas en el Journal Citation Reports (2018) en la categoría Education \& Educational Research, una sola se especializa en la temática de educomunicación (Comunicar), cuatro (Enseñanzas de las Ciencias, Porta Linguarum, RELIME y Movimento) son de temáticas muy específicas que usualmente no admiten estudios fuera de sus límites temáticos y alcances (lingüística, educación física, STEM, etc.), mientras que las cinco restantes ${ }^{4}$ permiten estudios de educación con temática más abierta, lo que evidencia con meridiana claridad una super-restricción de espacios selectivos y de impacto de publicación sobre educomunicación en nuestro idioma.

b) Scopus (Elsevier): al ser una base de datos que hace una medición temporal más amplia de las citas —en vez de dos años como el JCR, toman las citas recibidas de tres-, presenta una mayor cantidad de revistas científicas iberoamericanas en las categorías en estudio. De las 320 revistas emergentes en la categoría Communication, 14 son la-

\footnotetext{
${ }^{4}$ De estas cinco, International Journal of Educational Technology in Higher Education (Emerald Publishing) admite los artículos solo en idioma inglés.
} 
tinoamericanas (principalmente brasileras) y 15 españolas, totalizando 29 revistas que representan el 9,06\% de todo el universo; mientras que en la categoría Education emergen 1044 publicaciones de las que 35 son españolas y 39 latinoamericanas, totalizando 74 (7,08\%). Entre ambas categorías, integran una muestra de 103 revistas científicas iberoamericanas, un número muy superior a las 10 que suplen ambas categorías en el JCR.

Por supuesto, no todas las revistas publican estudios sobre educomunicación, para lo que resulta necesario realizar una búsqueda por descriptores y booleanos en esta base de datos, lo que nos daría como resultado las revistas que más publican sobre ello (ver figura 3 ).

Figura 3. Top 20 revistas iberoamericanas que más han publicado sobre educomunicación (Media Education) 2011-2019

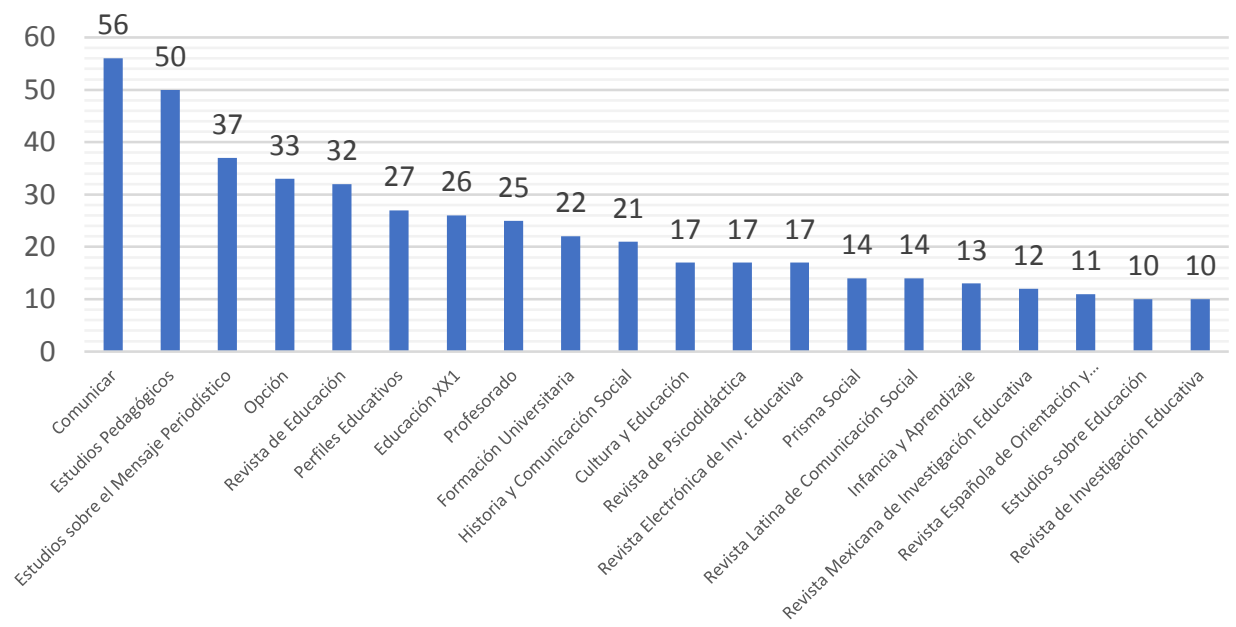

Fuente: elaboración propia.

La figura anterior refleja un liderazgo temático por parte de Comunicar, de España (Q1 en Educación, Comunicación y Estudios Culturales), seguido muy de cerca por Estudios Pedagógicos, de la Universidad Austral de Chile (Q3 en Educación) y Estudios sobre el Mensaje Periodístico, de la Universidad Complutense de Madrid (Q3 en Comunicación).

Si bien España se mantiene como el país con mayor tradición de publicaciones en la materia, revistas de otras latitudes como Opción, de la Universidad del Zulia de Venezuela (Q3 en Social Sciences y Arts and Humanities), Perfiles Educativos, de la Universidad Nacional Autónoma de México (Q3 en 
Educación), Formación Universitaria del Centro de Información Tecnológica de Chile (Q2 en Educación) o la Revista Mexicana de Investigación Educativa (Scopus Q3 en Educación), también se ubican en el top 20 de revistas de esta base de datos que más publican sobre educomunicación (Media Education).

\section{Publicaciones del Emerging Sources Citation Index (ESCI-Clarivate)}

El Emerging Sources Citation Index (Índice de Citas de Fuentes Emergentes) es un listado de revistas científicas producido desde 2015 a las que Clarivate Analytics les permite incluir sus artículos en la base de datos Web of Science (WoS). Este índice es fundamentalmente un "purgatorio» de revistas que esperan entrar en el exclusivo JCR, pero que da la posibilidad a publicaciones regionales y más pequeñas, no dependientes de grandes emporios editoriales, de posicionar sus contenidos en su buscador científico, lo que les facilita llegar a una audiencia más distintiva de investigadores de todo el mundo.

A diferencia del JCR y de Scopus, las revistas ingresan al ESCI por un estudio previo que realiza Clarivate Analytics sobre aspectos formales de la publicación, especialmente los que tienen que ver con transparencia editorial, revisión por pares, prácticas éticas, requerimientos técnicos e interoperabilidad, información bibliográfica, metadatos en inglés, entre otros. Para 2018/19, la categoría «Educación» del ESCI contaba con 486 revistas, de las cuales 65 son españolas, 27 brasileras, 14 colombianas, 4 mexicanas, 4 venezolanas, 4 argentinas, 4 ecuatorianas, 3 costarricenses, 3 cubanas, 3 peruanas, 2 chilenas; lo que totaliza en 133 revistas iberoamericanas Emerging en la categoría Educación, representando un $27,36 \%$ de todo el universo 5 .

En la siguiente tabla encontraremos las 10 primeras revistas iberoamericanas de la categoría «Educación» del Emerging Sources Citation Index, organizadas por posición, decil, factor de impacto y percentil:

Tabla 2. Top 10 Revistas Iberoamericanas de la categoría «Education» del Emerging Sources Citation Index 2018-2019

\begin{tabular}{|l|r|r|r|r|c|}
\hline \multicolumn{1}{|c|}{ Revista } & Posición & Decil & Impacto & Percentil & País \\
\hline $\begin{array}{l}\text { Journal of New Approaches in } \\
\text { Educational Research }\end{array}$ & 52 & D2 & 1,051 & 89 & España \\
\hline $\begin{array}{l}\text { RIED-Revista Iberoamericana de } \\
\text { Educación a Distancia }\end{array}$ & 56 & D2 & 1,017 & 88 & España \\
\hline Open Praxis & 64 & D2 & 1,000 & 86 & España \\
\hline Educar & 93 & D2 & 0,854 & 81 & España \\
\hline
\end{tabular}

5 Varias de estas publicaciones se encuentran indexadas en Scopus. 


\begin{tabular}{|l|r|r|r|r|c|}
\hline $\begin{array}{l}\text { Revista Latinoamericana de } \\
\text { Tecnología Educativa-RELATEC }\end{array}$ & 100 & D3 & 0,830 & 79 & España \\
\hline $\begin{array}{l}\text { Revista Eureka sobre Enseñanza } \\
\text { y Divulgación de las Ciencias }\end{array}$ & 136 & D3 & 0,702 & 72 & España \\
\hline $\begin{array}{l}\text { Latin American Journal of Content } \\
\text { \& Language Integrated-LACLIL }\end{array}$ & 138 & D3 & 0,692 & 71 & Colombia \\
\hline $\begin{array}{l}\text { Relieve-Revista Electrónica } \\
\text { de Investigación y Evaluación } \\
\text { Educativa }\end{array}$ & 139 & D3 & 0,692 & 71 & España \\
\hline $\begin{array}{l}\text { RIE-Revista de Investigación } \\
\text { Educativa }\end{array}$ & 144 & D3 & 0,683 & 70 & España \\
\hline $\begin{array}{l}\text { OCNOS-Revista de Estudios } \\
\text { sobre la Lectura }\end{array}$ & 154 & D4 & 0,625 & 68 & España \\
\hline
\end{tabular}

Fuente: elaboración propia a partir del Ranking ESCI de Revista Comunicar ${ }^{6}$.

Por su parte, igualmente para 2018/19, la categoría «Comunicación» del ESCI contaba con 114 revistas indexadas, de las cuales 25 son españolas, 4 brasileras, 3 argentinas, 3 chilenas, 1 colombiana, 1 ecuatoriana y 1 peruana, lo que totaliza 38 revistas iberoamericanas ${ }^{6}$, representando un 33,33\% del universo. Al igual que en el caso anterior, presentamos en la siguiente tabla el top 10 de revistas de Comunicación de este índice emergente:

Tabla 3. Top 10 Revistas Iberoamericanas de la categoría «Communication» del Emerging Sources Citation Index 2018-2019

\begin{tabular}{|l|r|r|r|r|c|}
\hline \multicolumn{1}{|c|}{ Revista } & Posición & Decil & Impacto & Percentil & País \\
\hline $\begin{array}{l}\text { Review of Communication } \\
\text { Research }\end{array}$ & 1 & D1 & 5,667 & 99 & España \\
\hline Cuadernos.info & 15 & D2 & 0,800 & 86 & Chile \\
\hline Communication \& Society & 19 & D2 & 0,699 & 83 & España \\
\hline $\begin{array}{l}\text { Revista Mediterránea } \\
\text { de Comunicación }\end{array}$ & 22 & D2 & 0,683 & 80 & España \\
\hline $\begin{array}{l}\text { Revista Latina de Comunicación } \\
\text { Social }\end{array}$ & 28 & D3 & 0,595 & 75 & España \\
\hline Palabra Clave & 36 & D4 & 0,464 & 68 & Colombia \\
\hline Comunicación y Hombre & 43 & D4 & 0,393 & 62 & España \\
\hline Index Comunicación & 46 & D5 & 0,357 & 60 & España \\
\hline $\begin{array}{l}\text { Anàlisi-Quaderns de Comunicació } \\
\text { i Cultura }\end{array}$ & 54 & D5 & 0,300 & 53 & España \\
\hline Trípodos & 60 & D6 & 0,256 & 47 & España \\
\hline
\end{tabular}

Fuente: elaboración propia a partir del Ranking ESCI de Revista Comunicar?.

6 Disponible en línea en: https://bit.ly/2DFDw1L (Fecha de consulta: 29/11/2019). 
El Emerging Source Citation Index ha venido ganando espacio como un indicador de calidad en diversas agencias de acreditación y en programas de doctorado - sobre todo en España-, por lo que resulta una «vía de escape» importante que tienen los investigadores hispanohablantes para la publicación. No en vano, solo en español hay 811 artículos publicados sobre educomunicación en la WoS bajo la categoría ESCI, siendo las revistas que más publican sobre el tema las siguientes:

Tabla 4. Top 10 Revistas Iberoamericanas del Emerging Sources Citation Index que más publican sobre educomunicación*

\begin{tabular}{|l|l|r|c|}
\hline \multicolumn{1}{|c|}{ Revista } & \multicolumn{1}{c|}{ Ámbito } & Arts. & País \\
\hline Revista Mediterránea de Comunicación & Comunicación & 23 & España \\
\hline Revista Conrado & Educación & 21 & Cuba \\
\hline Revista Latina de Comunicación Social & Comunicación & 21 & España \\
\hline Prisma Social & Cs. Sociales & 20 & España \\
\hline Pixel Bit. Revista de Medios y Educación & Comunicación y Educación & 19 & España \\
\hline Edmetic & Comunicación y Educación & 17 & España \\
\hline $\begin{array}{l}\text { Profesorado. Revista de Curriculum } \\
\text { y Formación del Profesorado }\end{array}$ & Educación & 15 & España \\
\hline $\begin{array}{l}\text { Dilemas contemporáneos Educación, } \\
\text { Política y Valores }\end{array}$ & Educación & 14 & México \\
\hline $\begin{array}{l}\text { Revista Latinoamericana de Tecnología } \\
\text { Educativa }\end{array}$ & Educación & 11 & España \\
\hline $\begin{array}{l}\text { Revista Virtual Universidad Católica } \\
\text { del Norte }\end{array}$ & Cs. Sociales & 11 & Colombia \\
\hline
\end{tabular}

*Nota: Se toman como referencia las revistas que más han publicado de 2016 a 2019 artículos con metadatos bibliográficos (título, resumen y palabras clave) «Media Education» (Educomunicación).

Fuente: elaboración propia.

\section{Publicaciones históricas no catalogadas o indexadas}

No todos los esfuerzos de difusión sobre educomunicación provienen de las revistas de «impacto» o indexadas, pues son diversas las plataformas que vienen trabajando sin descanso en la difusión de estudios e investigaciones empíricas en toda Iberoamérica sobre esta temática. Se intentará a continuación realizar un breve recorrido en orden cronológico, limitando este análisis a las cuatro revistas históricas más importantes de la región que han publicado manuscritos o han tenido monográficos específicos y recurrentes sobre educomunicación.

7 Disponible en línea en: https://bit.ly/2DFDw1L (Fecha de consulta: 29/11/2019). 
1) Chasqui (Ecuador). La revista de Comunicación con mayor recorrido histórico de Latinoamérica, Chasqui, nace en 1972 en el seno del Centro Internacional de Estudios Superiores de Comunicación para América Latina (CIESPAL), en Quito (Ecuador). Aunque ingresó hace poco en el Emerging Sources Citation Index, esta publicación es una referencia obligada para los investigadores de comunicación, estudios culturales y educación de la región. Algunos hitos importantes de la Comunicación en Latinoamérica han sido reflejados por esta revista, como la carta fundacional de la Federación Latinoamericana de Periodistas (FELAP) (Chasqui, no. 1, 1981), las declaraciones de México y de Quito sobre la libertad de información [del informe MacBride] (Chasqui, no. 1, 1981), o el documento de constitución de la Federación Latinoamericana de Facultades de Comunicación Social (FELAFACS) (Chasqui, no. 2, 1982). Prácticamente todos los históricos de la Comunicación y la Educación han publicado en esta revista, lo que la convierte en una de las publicaciones más importantes de la región y referencia obligatoria.

2) Comunicación. Estudios Venezolanos de la Comunicación (Venezuela). De la mano del Centro Gumilla, de la Universidad Católica Andrés Bello, nace esta publicación en 1975. Aunque al igual que Chasqui esta revista apunta más a los estudios de la Comunicación y los estudios culturales latinoamericanos, muchos de sus números han estado dedicados a la educomunicación. En esta revista encontramos artículos de autores de la talla de Jean Baudrillard, Michéle Mattelart, José Marques de Melo, Antonio Pasquali, Marcelino Bisbal, entre otros. Vale la pena acotar que esta revista publica entre 3 y 6 números al año, por lo que podría ser una de las más prolíficas de Iberoamérica.

3) Contratexto (Perú). La revista Contratexto nace en 1985. Editada por la Universidad de Lima, desde 2015 tiene periodicidad semestral, mientras que de 2005 a 2014 fue un anuario y antes de 2005 publicaba sin periodicidad. Entre los autores que han publicado en ésta se encuentran nombres de la talla de Jesús Martín Barbero, Eliseo Verón, Rosa María Alfaro, María Teresa Quiroz-Velazco, Erick Torrico, Marcelino Bisbal, Rafael Roncagliolo, Alejandro Piscitelli, entre otros.

4) Comunicação \& Educação (Brasil). Editada desde 1994, esta revista nace con el auspicio del Departamento de Comunicaciones y Artes de la Universidad de Sao Paulo, con el trabajo de históricos de la educomunicación como Maria Aparecida Baccega, Adilson Odair Citelli, Roseli Figaro e Ismar de Oliveira Soares. Actualmente, Comunicação \& Educação publica con periodicidad semestral. 


\section{Congresos de referencia}

Al igual que en los espacios referidos ut supra, resulta muy complejo listar los congresos más importantes sobre educomunicación en nuestro idioma sin dejar fuera otros de relevancia, sobre todo cuando este tipo de actividades científicas suelen variar de temáticas, mesas, simposios, grupos de trabajo, seminarios y ponencias invitadas. Teniendo esto en cuenta, se proponen a continuación algunos de estos eventos científicos, tomando en consideración su tradición temática y su recorrido histórico:

\subsection{Congresos Internacionales Red Alfamed}

La Red Interuniversitaria Euroamericana de Investigación sobre Competencias Mediáticas para la Ciudadanía (Alfamed) se constituye el 1 de octubre de 2014 en la Universidad de Huelva (España), bajo el liderazgo de Ignacio Aguaded. En la actualidad está compuesta por más de 172 investigadores de 17 países iberoamericanos y está avalada por instituciones como la Organización de Estados Iberoamericanos (OEI), la Asociación Universitaria Iberoamericana de Postgrados (AUIP), la Alianza para las Civilizaciones de la Organización de Naciones Unidas (UNAOC), Media In Action (MIA) y el Media and Information Literacy (MIL) de la UNESCO.

Los Congresos Internacionales de la Red Alfamed, celebrados de forma anual, son un espacio específico de divulgación y networking sobre educomunicación, comunicación y educación, competencia mediática y digital, entre otros aspectos relacionados y, para 2020, contará con su quinta edición:

- I Congreso Internacional Red Alfamed «Competencias mediáticas y Educación». Universidad Santiago de Cali (Colombia), del 12 al 13 de septiembre de 2016.

- II Congreso Internacional Red Alfamed «Competencias mediáticas y habilidades de Comunicación». Universidad Federal de Juiz de Fora (Brasil), del 23 al 25 de octubre de 2017.

- III Congreso Internacional Red Alfamed «Comunicación-Educación para el buen vivir». Universidad Católica Luis Amigó (Colombia), del 10 al 12 de octubre de 2018.

- IV Congreso Internacional Red Alfamed «Competencia digital: Del acceso al empoderamiento». Instituto Dominicano de Evaluación e Investigación de la Calidad Educativa (República Dominicana), del 29 al 31 de mayo de 2019. 
- V Congreso Internacional Red Alfamed. Centro Internacional de Estudios Superiores de Comunicación para América Latina (CIESPAL) en Quito (Ecuador).

\subsection{Congresos Ibercom de Assibercom}

El Congreso Iberoamericano de Comunicación (IBERCOM) nace como un movimiento académico en 1986 en Sao Paulo (Brasil), en el marco del Congreso Brasilero de Ciencias de la Comunicación, con la coordinación de José Marques de Melo y de Maria Immacolata Vassallo de Lopes. Sin embargo, no fue hasta 1998, durante su quinta edición (celebrada en Porto, Portugal), cuando se crea la Asociación Iberoamericana de Comunicación (ASSIBERCOM), siendo ésta hoy por hoy su matriz institucional. IBERCOM es quizás uno de los eventos más referentes de la región, no solo por su dilatada trayectoria y numerosos participantes, sino porque ha sido caldo de cultivo para la génesis del asociacionismo de investigadores iberoamericanos como el de la Asociación Latinoamericana de Investigadores de la Comunicación (ALAIC), la Federación de Asociaciones de Ciencias de la Comunicación de habla portuguesa (LUSOCOM), la Asociación Española de Investigación de la Comunicación (AE-IC) y, más reciente, la Confederación Iberoamericana de Asociaciones Científicas y Académicas de Comunicación (CONFIBERCOM).

El Congreso IBERCOM actualmente se celebra con periodicidad bianual, habiéndose presentado en noviembre de 2019 su XVI edición. En la siguiente tabla se verifica su recorrido histórico y geográfico:

Tabla 5. Congresos IBERCOM 1986-2019

\begin{tabular}{|l|l|l|c|l|l|}
\hline Año & \multicolumn{1}{|c|}{ Ciudad } & \multicolumn{1}{|c|}{ País } & Año & \multicolumn{1}{|c|}{ Ciudad } & \multicolumn{1}{|c|}{ País } \\
\hline $\mathbf{1 9 8 6}$ & Sao Paulo & Brasil & $\mathbf{2 0 0 6}$ & Sevilla & España \\
\hline $\mathbf{1 9 8 9}$ & Florianópolis & Brasil & $\mathbf{2 0 0 7}$ & Guadalajara & México \\
\hline $\mathbf{1 9 9 3}$ & Barcelona & España & $\mathbf{2 0 0 9}$ & Madeira & Portugal \\
\hline $\mathbf{1 9 9 7}$ & Santos & Brasil & $\mathbf{2 0 1 1}$ & Santa Cruz de la Sierra & Bolivia \\
\hline $\mathbf{1 9 9 8}$ & Oporto & Portugal & $\mathbf{2 0 1 3}$ & Santiago de Compostela & España \\
\hline $\mathbf{2 0 0 0}$ & Santiago de Chile & Chile & $\mathbf{2 0 1 5}$ & Sao Paulo & Brasil \\
\hline $\mathbf{2 0 0 2}$ & Maia-Porto & Portugal & $\mathbf{2 0 1 7}$ & Lisboa & Portugal \\
\hline $\mathbf{2 0 0 4}$ & La Plata & Argentina & $\mathbf{2 0 1 9}$ & Bogotá & Colombia \\
\hline
\end{tabular}

Fuente: elaboración propia.

\subsection{Congresos ALAIC}

La Asociación Latinoamericana de Investigadores en Comunicación (ALAIC) fue creada en 1978, como un organismo de representación cultural de América Latina para la UNESCO, bajo el liderazgo y la conducción inicial de Antonio 
Pasquali (Venezuela) y de Luis Ramiro Beltrán (Bolivia). Sin embargo, debido a los problemas económicos de la región para la década de 1980, esta institución se debilitó, perdiendo vigencia. No fue hasta 1988 cuando representantes de la Asociación Mexicana de Investigadores de la Comunicación (AMIC), de la Sociedade Brasileira de Estudos Interdisciplinares da Comunicação (Intercom) y del Centro Internacional de Estudios Superiores de Comunicación para América Latina (Ciespal) se reunieron para un relanzamiento de ALAIC en 1989, en el seno del II Congreso IBERCOM, nombrándose como presidente a José Marques de Melo.

Desde 1992 ALAIC realiza su Congreso, con periodicidad bianual y en varios países de América Latina (ver tabla 6):

Tabla 6. Congresos ALAIC 1992-2020

\begin{tabular}{|c|l|l|c|l|l|}
\hline Año & \multicolumn{1}{|c|}{ Ciudad } & \multicolumn{1}{|c|}{ País } & Año & \multicolumn{1}{|c|}{ Ciudad } & País \\
\hline $\mathbf{1 9 9 2}$ & Sao Paulo & Brasil & $\mathbf{2 0 0 8}$ & México & México \\
\hline $\mathbf{1 9 9 4}$ & Guadalajara & México & $\mathbf{2 0 1 0}$ & Bogotá & Colombia \\
\hline $\mathbf{1 9 9 6}$ & Caracas & Venezuela & $\mathbf{2 0 1 2}$ & Montevideo & Uruguay \\
\hline $\mathbf{1 9 9 8}$ & Recife & Brasil & $\mathbf{2 0 1 4}$ & Lima & Perú \\
\hline $\mathbf{2 0 0 0}$ & Santiago de Chile & Chile & $\mathbf{2 0 1 6}$ & México & México \\
\hline $\mathbf{2 0 0 2}$ & Santa Cruz & Bolivia & $\mathbf{2 0 1 8}$ & San José & Costa Rica \\
\hline $\mathbf{2 0 0 4}$ & La Plata & Argentina & $\mathbf{2 0 2 0}$ & Medellín & Colombia \\
\hline $\mathbf{2 0 0 6}$ & Sao Leopoldo & Brasil & & & \\
\hline
\end{tabular}

Fuente: elaboración propia.

\subsection{Congresos AE-IC}

La Asociación Española de Investigadores de la Comunicación (AE-IC) nace en 1982 como la Asociación para la Investigación en Comunicación (AIC), presidida por varios de sus miembros como Bernardo Díaz Nosty, Enrique Bustamante, Manuel Alonso o Jesús Timoteo. Sin embargo, no fue hasta 2006, con motivo del Congreso IBERCOM celebrado en Sevilla, cuando se funda lo que hoy es la AE-IC. Hasta la fecha, la AE-IC ha realizado 7 ediciones de su congreso, siendo éstas:

- 2008 - Santiago de Compostela.

- 2010 - Málaga.

- 2012 - Tarragona.

- 2014 - Bilbao.

- 2016 - Madrid.

- 2018 - Salamanca.

- 2020 - Valencia. 
Aunque este Congreso no presenta comúnmente grupos de trabajo (GT) o secciones temáticas específicas de educomunicación, sí se verifican, en el histórico de comunicaciones, investigaciones en este ámbito, así como participación de ponencias invitadas en esta línea.

\subsection{Congreso Internacional de Educación Mediática y Competencia Digital}

La Universidad de Valladolid, campus María Zambrano (Segovia), organiza este encuentro de saberes desde 2015, un espacio para el debate sobre la importancia y las tendencias en los sistemas educativos, influidos por el entorno digital y su constante evolución, especializado en Educación Mediática, Comunicación y Educación y Alfabetizaciones múltiples.

Dirigido por Alfonso Gutiérrez Martín y Agustín García Matilla, este Congreso aborda desde políticas públicas y sus aplicaciones prácticas, pasando por comparaciones de las vivencias en el entorno educativo formal, hasta temas como la educomunicación y habilidades para la vida.

Su tercera edición (2017) se realizó paralelamente con la Media Education Summit, organizada por el Centre for Excellence in Media Practice (CEMP) y MIL-UNESCO, lo que evidencia meridianamente la importancia global que ha venido consiguiendo esta actividad.

\subsection{Congresos Comunicación e Información Digital (CICID)}

El Grupo de Investigación en Comunicación e Información Digital de la Universidad de Zaragoza, dirigido por Carmen Marta Lazo, con la colaboración del Gobierno de Aragón y el Fondo Europeo de Desarrollo Regional (FEDER), realiza este encuentro bianual desde 2007.

Su objetivo principal es fomentar el debate sobre las dinámicas de la comunicación digital y su evolución, mediante simposios, mesas redondas y debates abiertos entre los participantes. Las grandes líneas de investigación están basadas en la comprensión del comportamiento de las audiencias, cómo interactúan en los entornos digitales, las tendencias referidas al emprendimiento en el área digital y el avance de las narrativas digitales, sin dejar de lado en todas sus ediciones el enfoque de la educomunicación y la competencia mediática y digital.

Desde ese planteamiento, este congreso busca dar respuesta a cómo la sabiduría digital puede contribuir a la comunicación inteligente, hacia dónde evoluciona el periodismo, la toma de decisiones empresariales y el propio concepto de la información. En definitiva, se trata de un espacio académico para el análisis, la reflexión y el intercambio de conocimiento hacia el sector 
profesional, invitando a participar a los especialistas de las distintas ciencias sociales referentes al ámbito digital para enriquecer el encuentro.

\subsection{Congreso Comunicación y Pensamiento}

La Facultad de Comunicación de la Universidad de Sevilla realiza, desde 2016, este congreso anual donde, en cada edición, se debate con destacados investigadores iberoamericanos que trabajan en temas de comunicación vinculados al desarrollo social, desde el periodismo, la publicidad, las relaciones públicas, las redes sociales y la comunicación corporativa.

Cada edición presenta temas que van desde la reflexión en torno al uso de internet para desentrañar sus secretos que influyen en el comportamiento de las personas y trasladar las conclusiones al público, hasta la comprensión del uso de los móviles como mecanismos imprescindibles en el proceso de interacción, tanto en los procesos educativos como comunicativos, impulsando el desarrollo de futuros proyectos de investigación.

El foco de este congreso está en brindar a los participantes las herramientas para comprender su entorno, influido por la tecnología y las tendencias digitales para así producir contenidos basados en las mejores prácticas. También se establece como meta detectar las repercusiones que existen entre las brechas generacionales al momento de usar los nuevos medios.

\section{Conclusión}

La investigación en educomunicación no es de nueva data, aunque los estudios bibliométricos arrojen que existe cierta «desfronterización» y transdisciplinariedad en sus abordajes, e incluso algún atisbo de «corporativismo excluyente» de esta temática en revistas nucleares (core) de la comunicación y la educación. Esta situación deja en meridiana evidencia que, aunque esta temática esté siendo objeto de estudio desde hace más de medio siglo, sigue siendo emergente por su continua transformación, dependiente - como es lógicode las propias dinámicas de nuestro ecosistema comunicativo.

En este sentido, quizás por esta realidad, resultan excesivamente restringidos los espacios de difusión de las investigaciones de educomunicación, en especial si apuntamos a publicar en nuestro idioma en revistas iberoamericanas de alto impacto, pues solo una revista (Comunicar) está especialmente dedicada a esta temática, lo que le permite mantenerse en el centro de influencia frente a otras publicaciones.

Este «cuello de botella» está siendo resuelto de forma paulatina por las revistas emergentes (ESCI), las cuales han ido cogiendo el testigo de la publicación de investigaciones de educomunicación y, a su vez, han ido ganando mayor referencialidad en este campo, como ocurre con la Revista Mediterrá- 
nea de Comunicación (España), la Revista Conrado (Cuba) y la Revista Latina de Comunicación Social (España), entre otras.

Sin embargo, no podemos olvidarnos de esas publicaciones que tienen más de 50 años en un arrojo constante de visibilización de la educomunicación, así como aquellos espacios científicos a los cuales, a través de este capítulo, se les rindió un homenaje, entendiendo que estos esfuerzos se realizan muchas veces sin los suficientes apoyos.

\section{Referencias bibliográficas}

BERGER, C.R. y CHAFEe, S. H. (2006). On Bridging the Communication Gap. Human Communication Research, 15(2), 311-318. doi.org/10.1111/j.1468-2958.1988.tb00187.x

CASTILLO, A. y CARRETÓN, C. (2010). Investigación en Comunicación. Estudio bibliométrico de las revistas de comunicación en España. Comunicación y Sociedad, 23(2), 289-327.

DE-FILIPPO, D. (2013). La producción científica española en Comunicación en WoS. Las revistas indexadas en SSCI (2007-12). Comunicar, 21(41), 25-34. doi.org/10.3916/C41-2013-02

GÓMEZ, I.; FERNÁNDEZ, M.T. y SEBASTIÁN, J. (1999). Analysis of the Structure of International Scientific Cooperation Networks through Bibliometric Indicators. Scientometrics, 44(3), 441-457. doi.org/10.1007/BF02458489

GONZÁLEZ-SAMÉ, H. (2019). La investigación en comunicación en estudios de Grado y Postgrado de Universidades de Ecuador y Bolivia: Visión comparativa del estado actual y prospectivas metodológicas [Tesis Doctoral]. Universidad de Huelva.

GonZÁLEZ-SAMÉ, H.; RomERO-RodRíGUEZ, L.M. y AGUADED, I. (2017). La investigación en comunicación en Latinoamérica: Una aproximación histórica (1950-2016). Historia y Comunicación Social, 22(2), 427-445. doi.org/10.5209/HICS.57853

LOTERO-ECHEVERRI, G.; ROMERO-RODRÍGUEZ, L.M. y PÉREZ-RodRíGUEZ, M.A. (2019). Tendencias de las publicaciones especializadas en el campo de la educomunicación y alfabetización mediática en Latinoamérica. Interface, 23(68), 180-193. doi.org/10.1590/Interface.180193

MANCINAS-CháVEZ, R.; RoMERo-RodRíGUEZ, L. M. y AGUADED, I. (2016). Problemas de la divulgación de las investigaciones en Comunicación en revistas de alto impacto en español. F@ro, 1(23), 241-258.

PIÑEIRO-NAVAL, V. y MoRAIS, R. (2019). Estudio de la producción académica sobre comunicación en España e Hispanoamérica. Comunicar, 27(61), 113123. doi.org/10.3916/C61-2019-10 
Romero-RoDRíGUEZ, L. y AGUADED, I. (2016). Consumption of information and digital competencies of journalism students from Colombia, Peru and Venezuela. Convergencia Revista de Ciencias Sociales, (70), 1-20. 